\title{
Syphilis on the rise again in Germany - results from surveillance data for 2011
}

V Bremer (bremerv@rki.de) ${ }^{1}$, U Marcus ${ }^{1}, 0$ Hamouda ${ }^{1}$

1. Division for HIV/AIDS, STI and Blood-borne Infections, Department for Infectious Disease Epidemiology, Robert Koch Institute, Berlin, Germany

Citation style for this article:

Bremer V, Marcus U, Hamouda O. Syphilis on the rise again in Germany - results from surveillance data for 2011. Euro Surveill. 2012;17(29):pii=20222. Available online: http://www.eurosurveillance.org/ViewArticle.aspx?Articleld=2022

Article submitted on 9 July 2012 / published on 19 July 2012

In Germany, syphilis is notified anonymously. In 2011, 3,698 cases (incidence 4.5/100,000 inhabitants) were notified, an increase of $22 \%$ over 2010 . The increase was higher in men (23\%) than women (13\%) and $94 \%$ of the cases were male. Information on the possible way of transmission was available for $72 \%$ of cases. Of these, $84 \%$ were men who have sex with men, who seem to play a major role in the renewed increase in syphilis cases.

Syphilis had become a rare disease in Germany in the 1990s. In 2001, the surveillance system changed and laboratories in Germany were required to notify each new syphilis diagnosis directly to the Robert Koch Institute in Berlin [1]. Before 2001, syphilis was notifiable according to the Geschlechtskranheitengesetz (Sexually Transmitted Diseases Act). Physicians were asked to report clinical syphilis cases and no case definition was used. The number of syphilis cases doubled between 2001 and 2004 and reached 3,364 [2]. This increase between 2001 and 2004 was only observed among men, many of them men who have sex with men (MSM). Since the increase was only seen among men, we assume it was not an effect due to the new surveillance system. Notifications then remained stable until 2008, decreased in 2009 to 2,742 [3], and rose again to 3,033 in 2010 [4], an increase of $10.6 \%$. This report describes how syphilis cases have increased in Germany in 2011, in comparison to previous years.

\section{Syphilis surveillance}

In Germany, laboratories notify syphilis diagnoses directly and anonymously to the Robert Koch Institute. Physicians are required to complete the laboratory findings with clinical information. To identify possible double notifications, each incoming notification form is compared to previous notifications with regard to the month and year of birth of the case and the first three digits of the postal code of their place of residence. Since the notifications are completely anonymous and not identified by a code, potential double notifications are compared using the parameters date of diagnosis, antibody titres and reported clinical information, to differentiate between follow-up tests and new clinical episodes.
We used the following case definition:

- direct detection of Treponema pallidum by microscopic examination of fluid or smears from lesions, histological examination of tissues,

- or detection of antibodies against T. pallidum by screening test (T. pallidum haemagglutination assay (TPHA), T. pallidum particle agglutination assay (TPPA) or enzyme immunoassay (EIA)), confirmed by fluorescent treponemal antibody absorption (FTA$A B S$ ) or IgG immunoblot,

and

- venereal disease research laboratory test (VDRL) titre $>4$ (rapid plasma reagin $>8$ ),

- or VDRL titre >o and $<8$, and clinical information consistent with primary syphilis,

- or detection of treponemal IgM antibodies (by IgM enzyme-linked immunosorbent assay (ELISA), IgM immunoblot or 19S(IgM) FTA-ABS).

We described syphilis cases by month of diagnosis or notification (in case date of diagnosis was missing), age, sex and residence. Where such information was available, we analysed the data by transmission category and country of infection and origin.

\section{Results}

In 2011, 3,698 syphilis cases were notified, an increase of $22 \%$ over the 3.033 cases in 2010 . The observed increase was higher in men (23\%) than in women (13\%), and $94 \%$ of the cases were male (Figure 1). The overall incidence was 4.5 per 100,000 inhabitants (Figure 2).

The incidence in men was 14 times higher than in women (8.6 versus 0.6 per 100,000 inhabitants). The incidence rose in all age groups for men, while there was only a small increase in some age groups among women (Figure 3). The highest incidence in men $(19.1 / 100,000)$ was observed in the 30-39 year-olds, and the highest incidence in women $(1.7 / 100,000)$ in the 25-29 year-olds. 


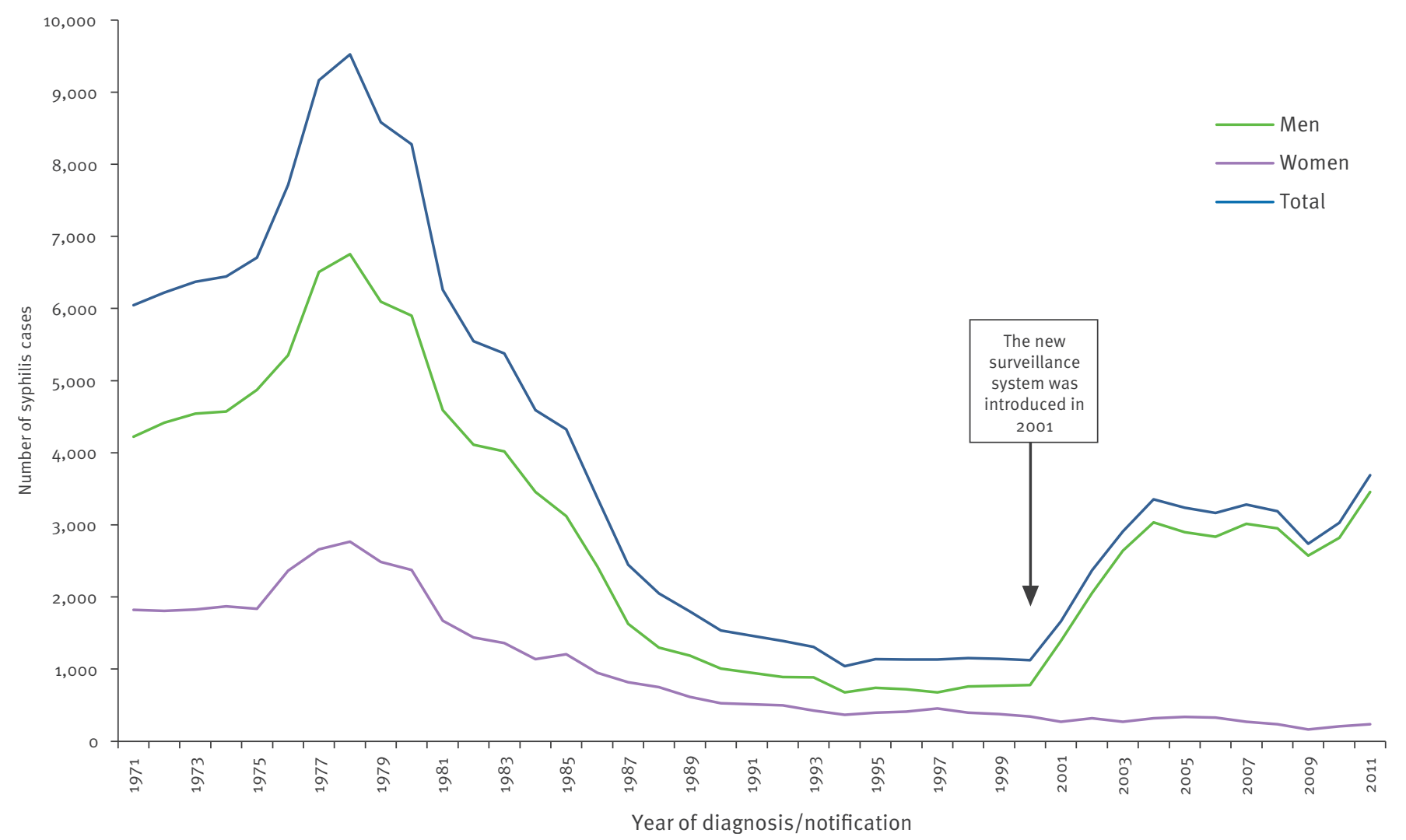

We used month of notification in case the date of diagnosis was missing.

Notification data according to the Sexually Transmitted Diseases Act (before 2001) and the Infection Protection Act (after 2001).

Information on transmission category was available for 2,645 cases $(72 \%)$ of the cases. In $84 \%$ of those, sex between men was mentioned as the probable route of transmission. Transmission through heterosexual contacts was mentioned in $16 \%$ of the cases. The largest increase in syphilis cases was observed in MSM, followed by cases without information on route of transmission and cases with probable heterosexual transmission. Information on country of infection was available for 2,659 cases (73\%). Of those, $93 \%$ acquired their infection in Germany. Infections acquired in western Europe were mostly in MSM, while heterosexual transmission prevailed in infections acquired in central or eastern Europe. Also, two cases of congenital syphilis were registered.

An increased incidence was observed in 11 of the 16 federal states, while it was stable in and decreased slightly in three federal states. The highest incidences were seen in the cities of Cologne (24.0/100,000), Frankfurt (21.0/100,000) and Berlin (18.0/100,000). Furthermore, we observed an unusual upsurge of syphilis cases among women and heterosexual men in the city of Dortmund in North Rhine-Westphalia and the surrounding area. The number of notified syphilis cases in women residing in Dortmund increased from two in 2009 to 10 in 2010 and 23 in 2011. At the same time, the number of notified syphilis cases in heterosexual men increased from eight in 2010 to 18 in 2011. Sex work or contact with sex workers was indicated as a possible way of transmission in nine of these cases. However, the available information was too incomplete to conclude that this outbreak was solely linked to sex work.

\section{Discussion}

We observed a considerable increase in notified syphilis cases in Germany in 2011. It has been the year with the highest number of notified cases since the introduction of the Infection Protection Act (Infektionsschutzgesetz) in 2001. Such a high level has not been observed since 1986, although comparability between surveillance data before and after 2001 is limited due to introduction of a case definition and a different reporting system. It is too early to know whether this is just a temporary rise or a new trend. However, we had already observed a moderate increase in notifications between 2009 and 2010. Since the notified syphilis cases continued to increase during the first three months of 2012, it is possible that a further increase in the number of syphilis cases in 2012 will be observed. 


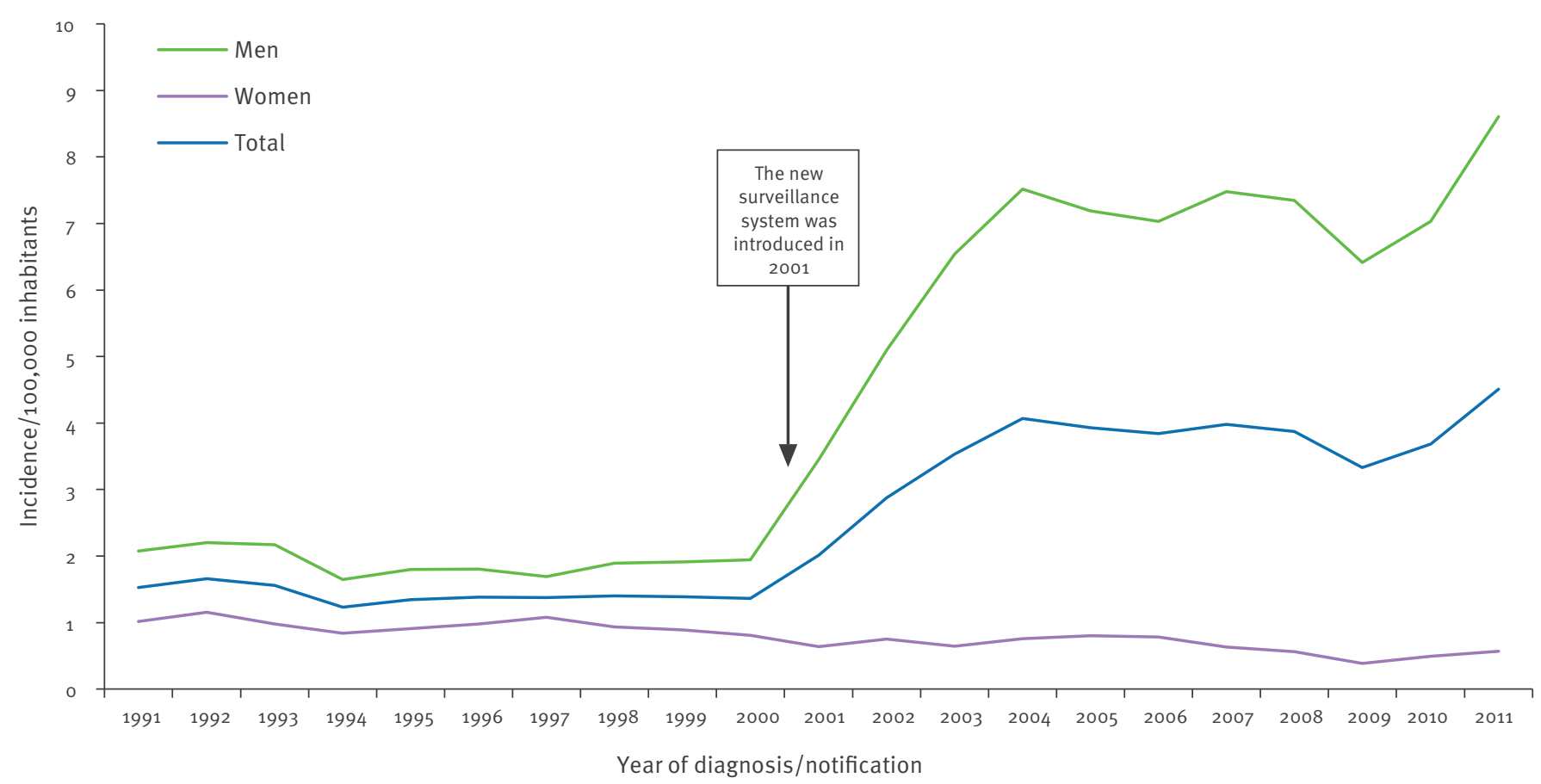

We used month of notification in case the date of diagnosis was missing.

Notification data according to the Sexually Transmitted Diseases Act (before 2001) and the Infection Protection Act (after 2001).

An increase in syphilis has been observed in several countries in western Europe between 1998 and $2005[5,6]$. Many of these syphilis cases were among MSM residing in large cities $[7,8]$. This has also been observed in Germany. Most syphilis cases among German MSM acquired their infection in Germany, which indicates that transmission is mainly occurring within the country. Part of the increase in cases among MSM could be explained by the inclusion of syphilis testing into the regular monitoring of human immunodeficiency virus (HIV)-positive MSM and a higher demand by HIV-negative MSM to get screened for sexually transmitted infections (STIs).

Until 2008, simultaneous increases and decreases of syphilis notifications in MSM in different regions had been observed. Following the country-wide decrease in syphilis case notifications in 2009 , also the increase in 2011 in the MSM population seems to be occurring in most regions in Germany. From behavioural studies among MSM - the last larger survey (European MSM Internet Survey; EMIS) was conducted in 2010 [9] there are no indications of any significant behavioural changes. Longer term trends towards increasing partner numbers and high levels of HIV serosorting (choosing sexual behaviour based on HIV status) particularly among MSM diagnosed with HIV may generally favour the spread of syphilis [10], but would not be sufficient to explain short term increases. Since undetected syphilis infections can increase the risk of HIV transmission [11], early diagnosis and treatment are important to minimise this risk. MSM with multiple partners should therefore be offered regular screening for syphilis and other STIs.

Although the large majority of cases in 2011 were observed among MSM, outbreaks among heterosexuals do occur. In Dortmund, we were not able to verify a possible link to sex work for the outbreak. Still, the local health authorities started to reinstate STI counselling and testing, aimed at sex workers. Private practitioners were informed about the outbreak. In 2012, only few syphilis cases among women and heterosexual men have so far been registered in Dortmund.

The increase of syphilis in MSM between 1998 and 2005 was observed in several European countries at the same time [6]. After that, surveillance data showed that the incidence of syphilis remained stable or declined in several western European countries, leading to an overall decrease of $7 \%$ in reported cases between 2006 and 2009 [12]. Since increases or decreases seem to be synchronised in several countries, it is possible that the recent development in Germany will be mirrored also in other western European countries. 


\section{FIGURE 3}

Incidence per 100,000 men and women of notified syphilis cases by age groups and year of notification or diagnosis, Germany, 1991-2011

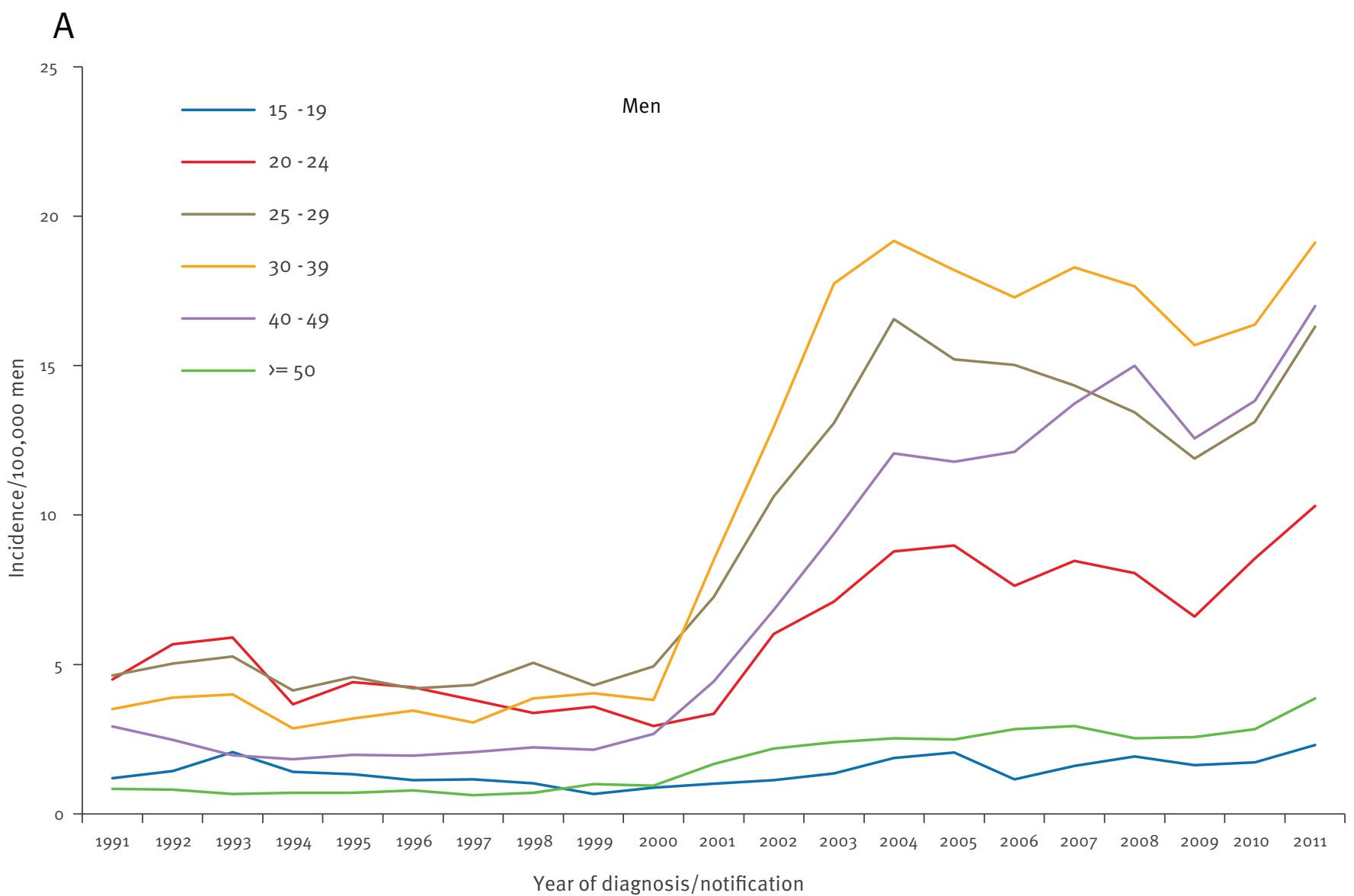

B

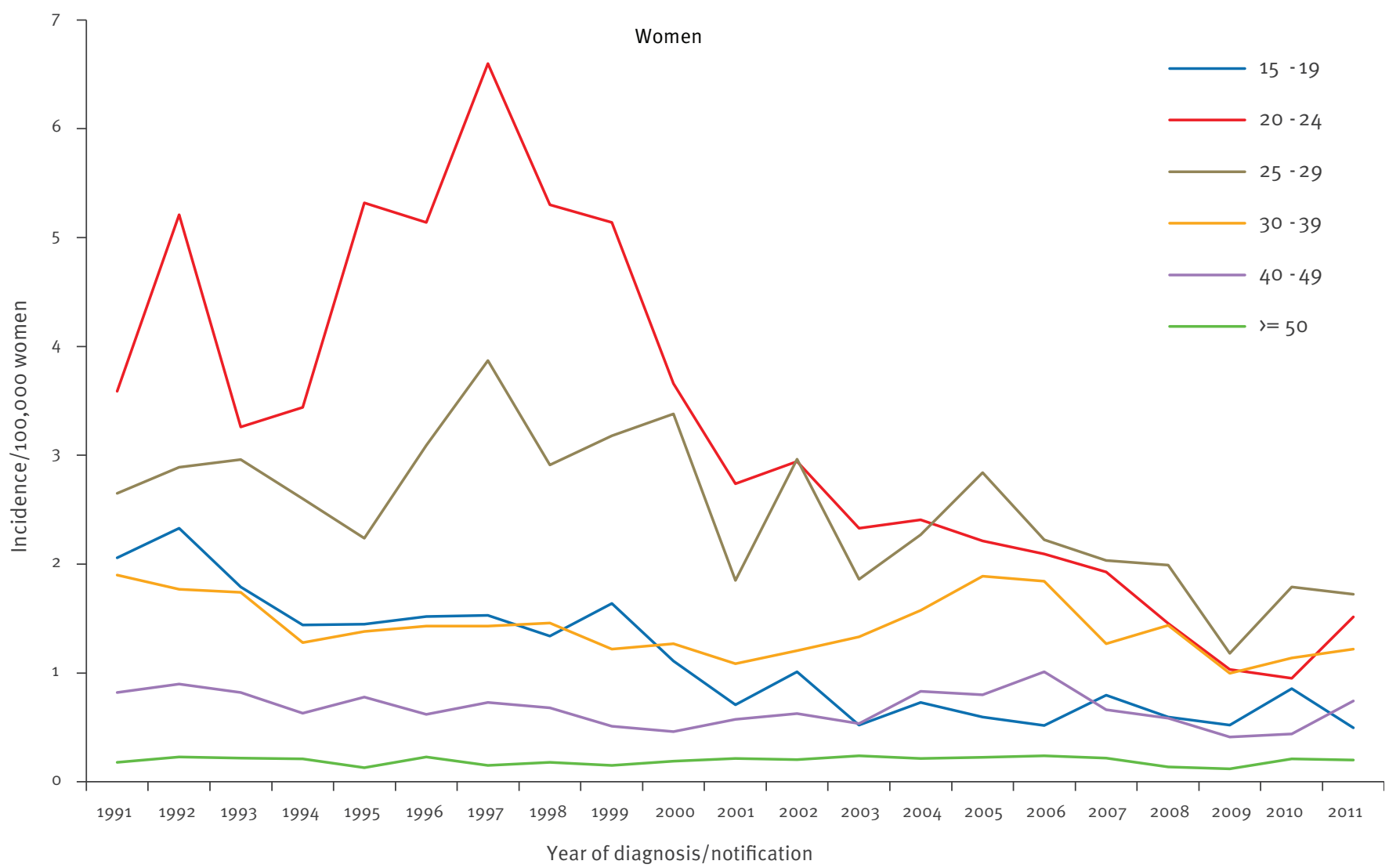

We used month of notification in case the date of diagnosis was missing.

Notification data according to the Sexually Transmitted Diseases Act (before 2001) and the Infection Protection Act (after 2001). 


\section{References}

1. Bremer V. Erneuter Anstieg der Syphilis-Meldungen in 2011. [New rise in syphilis notifications in 2011]. Epid Bull. 2011;24: 221-3. German. Available from: http://www.rki.de/ DE/Content/Infekt/EpidBull/Archiv/2012/Ausgaben/24_12. pdf?__blob=publicationFile

2. Marcus U, Hamouda O. Syphilis in Germany, 2004: diagnoses increasing, particularly in smaller cities and rural areas. Euro Surveill. 2005;10(30):pii=2759. Available from: http://www. eurosurveillance. 0 rg/ViewArticle. aspx? Articleld=2759

3. Marcus U. Syphilis in Deutschland im Jahre 2009. [Syphilis in Germany in 2009]. Epid Bull. 2010;49:487-91. German.

4. Robert Koch Institute (RKI). Infektionsepidemiologisches Jahrbuch meldepflichtiger Krankheiten für 2010 [Annual epidemiological report on notifiable diseases for 2010]. Berlin:RKI; 2011. German. Available from: http://www. rki.de/DE/Content/Infekt/Jahrbuch/Jahrbuch_2010. pdf?_blob=publicationFile

5. Nicoll A, Hamers FF. Are trends in HIV, gonorrhoea, and syphilis worsening in western Europe? BMJ. 2002;324(7349):1324-7.

6. Savage EJ, Hughes G, Ison C, Lowndes CM, the European Surveillance of Sexually Transmitted Infections (ESSTI) network. Syphilis and gonorrhoea in men who have sex with men: a European overview. Euro Surveill. 2009;14(47):pii=19417. Available from: http://www. eurosurveillance.org/ViewArticle. aspx?Articleld=19417

7. Cowan SA. Syphilis in Denmark-Outbreak among MSM in Copenhagen, 2003-2004. Euro Surveill. 2004;9(12):pii=498. Available from: http://www.eurosurveillance.org/ViewArticle. aspx?Articleld $=498$

8. Vall Mayans M, Sanz Colomo B, Armengol P, Loureiro E. Outbreaks of infectious syphilis and other STIs in men who have sex with men in Barcelona, 2002-3. Euro Surveill. 2004;8(44):pii=2578. Available from: http://www. eurosurveillance.org/ViewArticle.aspx?Articleld $=2578$

9. EMIS. The European MSM Internet Survey. Berlin: Robert Koch Institute. Available from: www.emis-project.eu

10. Marcus U, Schmidt AJ, Hamouda O. HIV serosorting among HIV-positive men who have sex with men is associated with increased self-reported incidence of bacterial sexually transmissible infections. Sex Health. 2011;8(2):184-93

11. Funnye AS, Akhtar AJ. Syphilis and human immunodeficiency virus co-infection. J Natl Med Assoc. 2003;95(5):363-82

12. European Centre for disease prevention and Control (ECDC). Annual Epidemiological Report. Reporting on 2009 surveillance data and 2010 epidemic intelligence data. Stockholm: ECDC; 2011. Available from: http://www.ecdc.europa.eu/en/ publications/Publications/1111_SUR_Annual_Epidemiological_ Report_on_Communicable_Diseases_in_Europe.pdf 